\title{
TIME RESOLVED OBSERVATION OF RESONANT AND NON-RESONANT CONTRIBUTIONS TO THE NONLINEAR SUSCEPTIBILITY $\chi^{(3)}$
}

\author{
W. ZINTH, A. LAUBEREAU * and W. KAISER \\ Physik Department der Technischen Universität München, München, West Germany
}

Received 19 June 1978

The resonant and nonresonant part of $\chi^{(3)}$ are distinguished by their different time behavior. The medium is coherently excited by two picosecond light pulses of defined frequency difference and the state of the system is monitored by a third properly delayed probe pulse. Results are presented on neat liquids of carbontetrachloride and cyclohexane and on the mixture of $\mathrm{CCl}_{4}: \mathrm{C}_{6} \mathrm{H}_{12}$.

\section{Introduction}

In recent years, the optical third order nonlinear susceptibility $\chi^{(3)}$ has attracted considerable attention. Several three-wave mixing techniques have been developed to determine $\chi^{(3)}$ coefficients [1-5]. Various contributions to the nonlinear susceptibility originating from single frequency resonances [6], Raman (difference frequency) resonances and two-photon resonances were inferred from measurements of the frequency dependence of $\chi^{(3)}$ and/or the polarisation properties $[3,5]$ of the applied light fields. Nonlinear refractive indices far from resonance were measured by interferometric techniques and by self-phase modulation [7, 8]. Most experiments were made under quasi-stationary conditions using light pulses of a duration of several $10^{-9} \mathrm{~s}$.

On the other hand, there exist extensive investigations of light-matter interactions performed under transient conditions [9-12]. The properties of the coherent material excitation were measured as a function of time yielding new information on the dephasing time $T_{2}$ and the inhomogeneous broadening of the molecular transition. In these experiments, the resonant excitation of strong Raman lines in pure systems (liquids, gases) was studied; the resonant part of $\chi^{(3)}$ dominates and the other (nonresonant) contributions were negligible.

\footnotetext{
* Present address: Physikalisches Institut Universität Bayreuth, Bayreuth, Germany.
}

In this letter we wish to demonstrate that resonant and nonresonant parts of $\chi^{(3)}$ can be investigated by time resolved techniques. Coherent Raman probe scattering of the pure liquids $\mathrm{CCl}_{4}$ and $\mathrm{C}_{6} \mathrm{H}_{12}$ and of the mixture $\mathrm{CCl}_{4}: \mathrm{C}_{6} \mathrm{H}_{12}$ is studied. We use a three-pulse system which allows measurements of coherent probe scattering in practically any medium. Starting with a chirp-free single picosecond light pulse with frequency $\omega_{L}$ we produce a synchronized pulse at a Stokes shifted frequency $\omega_{\mathrm{S}}$ via transient stimulated Raman scattering in a first generator cell. Both pulses at frequency $\omega_{\mathrm{L}}$ and $\omega_{\mathrm{S}}$ travel through a second medium (sample) where they excite the material coherently. The time behavior of the excitation is monitored by a properly delayed third pulse measuring the coherent Stokes scattering signal.

\section{Theory}

The build-up of the Stokes pulse in the generator by transient stimulated Raman scattering has been described in earlier publications $[11,14]$. Here, we focus our attention on the probing process.

The propagation of light pulses and the interaction with the surrounding medium is represented by the nonlinear wave equation

$\Delta E-\frac{1}{c^{2}} \frac{\partial^{2}}{\partial t^{2}}\left(\mu^{2} E\right)=\frac{4 \pi}{c^{2}} \frac{\partial^{2}}{\partial t^{2}} P^{\mathrm{NL}}$,

$E$ denotes the total electric field, $\mu$ is the optical index 
of refraction. The nonlinear polarisation $P$ NI. acacounts for the interaction of the light ficld with the material excitation. Close to a Raman (difference frequency) resonance we may separate $P^{\mathrm{N} l}$, into two parts: (i) A resonant part due to the molecular transition of interest with Raman polarisability $\partial \alpha / \partial q$ and (ii) a non-resonant contribution of the electronic levels which is represented by the non-resonant part $\overleftrightarrow{\chi}_{N K}^{(3)}$ of the third order nonlinear susceptibility. Thus we write

$P^{\mathrm{NL}}=P_{\mathrm{R}}^{\mathrm{NI}}+P_{\mathrm{NR}}^{\mathrm{NL}}=N \frac{\partial \alpha}{\partial q}\langle q\rangle E+\overleftrightarrow{\chi}_{\mathrm{NR}}^{(3)} E E E$.

$N$ is the number density of the molecules. $\langle q\rangle$ denotes the expectation value of the displacement $q$ connected with the molecular transition. For the vibrational transition considered here $q$ is the normal mode operator. For simplicity we take the Raman polarisability $\partial \alpha / \partial q$ to be a scalar; i.e. a highly polarized Raman line is investigated. It should be noted that for the picosecond light fields discussed here the nonresonant part $\boldsymbol{P}_{\mathrm{NR}}^{\mathrm{NL}}$ has the same form as for longer light pulses. This fact results from the extremely short time constants of the electronic contributions to $\chi_{\mathrm{NR}}^{(3)}$ of the order of $10{ }^{14} \mathrm{~s}$. The transient response of the resonant part. on the other hand, has to be taken into account via the coherent amplitude $\langle q\rangle|13|$ which obeys the equation of motion $|11|$ :

$$
\frac{\partial^{2}}{\partial t^{2}}\langle q\rangle+\frac{2}{T_{2}} \frac{\partial}{\partial t}\langle q\rangle+\omega_{0}^{2}\langle q\rangle=\frac{1}{2 m}-2 \bar{n} \cdot \frac{\partial \alpha}{\partial q} E^{2} .
$$

$\omega_{0}$ represents the transition frequency, $T_{2}$ the dephasing time, $m$ the reduced mass of the molecular vibration. $n$ is the equilibrium occupation probability of the first excited vibrational level. For the mode investigated here we have $\ddot{n} \ll 1$.

To solve eqs. (1) (3) we make the ansatz of plane waves for the electric field and the resonant material excitation propagating in the $x$-direction.

$E=\frac{1}{2}\left\{\sum_{j} E_{j} \exp \left(\mathrm{i} k_{j} x \quad \mathrm{i} \omega_{j} t\right)+\right.$ c.c. $\}$

$q=\frac{1}{2} Q \exp \left(\mathrm{i} k_{\mathrm{v}} x-\mathrm{i} \omega_{\mathrm{v}} t\right)+\mathrm{c.c}$.

The total light field consists of the incident laser and Stokes fields. $E_{L}, E_{\mathrm{S}}$ (frequencies $\omega_{\mathrm{L}}, \omega_{\mathrm{S}}$ ), the delayed probing field $E_{\mathrm{L} 2}$ and the scattered field $E_{\mathrm{S} 2}$ (frequencies $\left.\omega_{\mathrm{L} 2}, \omega_{\mathrm{S} 2}\right)$. The frequency and wave vector of the material excitation is $\omega_{v}=\omega_{L}-\omega_{S}$ and $k_{v}=$
$k_{\mathrm{L}}-k_{\mathrm{S}}$, respectively. We consider specilic polat isation conditions: The incident laser and Stokes fields are assumed to be linearly polarized in the $y$-direction while the fieldis $E_{1.2}$ and $E_{\mathrm{S} 2}$ point in the z-direction. This choice allows to distinguish the Stokes fields of the excitation and probing process. The distinction is of particular importance for the case of equal frequencics $\omega_{\mathrm{I}}=\omega_{\mathrm{L} 2}$ and correspondingly $\omega_{\mathrm{S}}=\omega_{\mathrm{S} 2}$. The relevant tensur element of $\overleftrightarrow{\chi}_{N R}^{(3)}$ for coherent Stokes scattering with our polarisation conditions is $\chi_{\mathrm{NR}, z y z y}^{(3)}$;it will be abbreviated by $\chi_{\mathrm{NR}} \cdot \chi_{N_{\mathrm{R}}}$ is a real quantity to a good approximation. Since $\langle q\rangle$ and $\partial \alpha / \partial q$ are scalars here (isotropic Raman scattering). the resonant part of $p^{\mathrm{NL}}$ is not affected by our polarisation conditions. Introduction of eq. (4) in eqs. (1) and (2) and neglecting second order derivatives of the slowly varying amplitudes yields the expression

$$
\begin{aligned}
& \left(\frac{\partial}{\partial x}+\frac{1}{v_{\mathrm{S} 2}} \frac{\partial}{\partial t}\right) E_{\mathrm{S} 2}={\stackrel{\pi \omega_{\mathrm{S} 2}^{2}}{{ }^{2} k_{\mathrm{S} 2}}\left\{N(\partial \alpha / \partial q) Q^{*} E_{12}\right.}^{\left.+3 \chi_{\mathrm{NR}} E_{\mathrm{L}}^{*} E_{\mathrm{S}} E_{\mathrm{L} 2}\right\} \exp (\mathrm{i} \Delta k x),}
\end{aligned}
$$

where $\Delta k=k_{\mathrm{S} 2}-k_{\mathrm{L} 2}+k_{\mathrm{v}}$ is the $k$-mismatch of the probe process: $k_{\mathrm{L} 2}$ and $k_{\mathrm{S} 2}$ denote wave vectors of the probe pulse and the scattered Stokes ficld, respectively.

Coupling terms of the Stokes probe scattering by the pump and probe pulses have been neglected in eq. (5). This approximation is well justified for the experiments discussed below.

In experimental investigations with picosecond pulses we observe the time integrated scattering signal $\int\left|F_{\mathrm{S}_{2}}\right|^{2} \mathrm{~d} t$ as a function of the time delay $t_{1}$ between the incident punp fields and the probe pulse. Integrating eq. (5) we obtain

$$
\begin{aligned}
& S^{\operatorname{coh}\left(t_{D}\right)}=\left|\begin{array}{ll}
\pi \omega_{S 2}^{2} & \sin (\Delta k l / 2) \\
c^{2} k_{S 2} & \cdots k l / 2
\end{array}\right|^{2} \\
& \times \int_{-\infty}^{\infty}\left(\mathrm{d} t\left|F_{\mathrm{L} 2}\left(t \cdots t_{\mathrm{D}}\right)\right|^{2}\right. \\
& \times\left|N\left(\partial \alpha_{i} / \partial q\right) Q^{*}(t)+3 \chi_{\mathrm{NR}} E_{\mathrm{L}}^{*}(t) E_{\mathrm{S}}(t)\right|^{2} .
\end{aligned}
$$

Here $l$ denotes the sample length. The coherent amplitude $Q$ entering eq. (6) is calculated from cq. ( 3 ). It is interesting to see that the scattering signal $S^{\mathrm{coh}}$ depends strongly on the mismatch $\Delta k$. Maximum scattering occurs for $\Delta k=0$. The time evolution of $S^{\operatorname{coh}}\left(t_{D}\right)$ 
is determined by $Q^{*}(t)$ and $E_{\mathrm{L}}^{*}(t) E_{\mathrm{S}}(t)$ according to the respective weighting factors $N(\partial \alpha / \partial q)$ and $3 \chi_{\mathrm{NR}}$.

Numerical results of $S^{\mathrm{coh}}\left(t_{\mathrm{D}}\right)$ calculated with the help of eqs. (3) and (6) are presented in fig. 1. A gaussian time dependence and equal pulse duration $t_{\mathrm{p}}$ are assumed for the incident laser field $E_{\mathrm{L}}$ and for the probe pulse $E_{\mathrm{L} 2}$. The incident Stokes pulse (generated in a preceding Raman generator) is evaluated from the theory of transient stimulated Raman scattering [11]. The scattered Stokes signal is plotted as a function of delay time $t_{\mathrm{D}} / t_{\mathrm{p}}$. The three solid curves are calculated for a dephasing time $T_{2} / t_{\mathrm{p}}=1.2$ and for different ratios of $\chi_{\mathrm{NR}} / N(\partial \alpha / \partial q)$. For $\chi_{\mathrm{NR}} \rightarrow 0$ one calculates curve 1 . This situation has been investigated previously [10]. The coherent signal rises rapidly to a delayed maximum at $t_{\mathrm{D}} / t_{\mathrm{p}} \approx 1$. For larger delay times the signal decreases exponentially with the dephasing time $T_{2} / 2$.

For diluted systems and/or weak Raman lines the

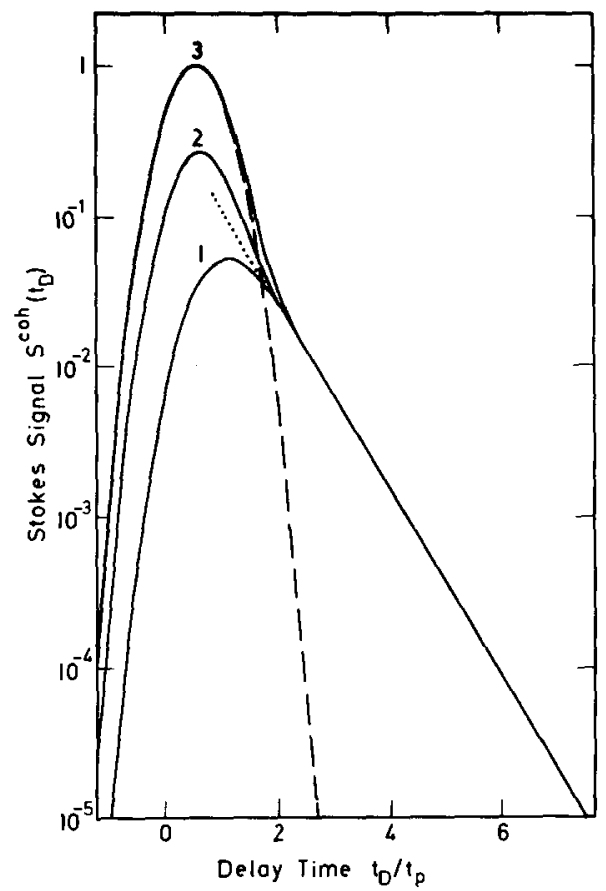

Fig. 1. Calculated coherent probe scattering signal $S^{\mathrm{coh}}$ as a function of delay time $t_{\mathrm{D}} / t_{\mathrm{p}}$. A gaussian time dependence of the incoming laser pulse (duration $t_{\mathrm{p}}$ ) was used. The curves are calculated for $T_{2} / t_{\mathrm{p}}=1.2$ and values $\chi_{\mathrm{NR}} / N(\partial \alpha / \partial q)=0$, $0.3,0.6$ (curves 1 to 3 ) and for a purely nonresonant susceptibility (broken line). The dotted line extends the exponential slope of $T_{2} / 2$. finite contribution of $\chi_{\mathrm{NR}}$ has to be considered. Curves 2 and 3 are calculated for values of $\chi_{\mathrm{NR}} / N(\partial \alpha / \partial q)=$ 0.3 and 0.6 , respectively. The different time behavior of the scattering signal around the maximum should be noted. The maximum is shifted to larger signal values and to somewhat shorter times. The nonresonant contribution decays rapidly in a first step. Then the signal curve turns to an exponential decay with slope $T_{2} / 2$. In the latter region, the nonresonant contribution has disappeared and the resonant part with finite lifetime $T_{2}$ prevails. The dotted line extends the slope to smaller values of $t_{\mathrm{D}}$.

Of interest is the broken curve in fig. 1. It is calculated for the case where we are far from resonance; i.e. for $\partial \alpha / \partial q=0$ (with the same parameters as curve 3). A rapid non-exponential decay of the coherent signal is obtained. The shape of this curve represents the convolution of the proving pulse $E_{\mathrm{L} 2}^{2}$ with the nonresonant excitation $\propto\left|E_{\mathrm{L}} E_{\mathrm{S}}^{*}\right|^{2}$. Since the Stokes pulse generated by transient stimulated scattering is shorter than the laser pulse by a factor of two $[14,11]$, the signal curve represents - to a good approximation the shape of the incident probe pulse. Coherent probe scattering under nonresonant excitation conditions offers an attractive possibility for the experimental determination of picosecond and subpicosecond pulse shapes.

The maximum amplitude of the broken curve is proportional to $\chi_{\mathrm{NR}}^{2}$ while curve 1 (purely resonant excitation) is determined by $(N \partial \alpha / \partial q)^{2}$. Comparing experimental data for both situations allows to deduce the value of $\chi_{\mathrm{NR}}$ from the Raman gain factor or from the spontaneous scattering cross section.

\section{Experimental}

Our experimental set-up is depicted schematically in fig. 2. A single chirp-free picosecond laser pulse from a $\mathrm{Nd}$ : glass laser system [15] is frequency-doubled in a KDP crystal to $\tilde{v_{L}}=18995 \mathrm{~cm}^{-1}$. The pulse of duration 5.2 ps passes a $\lambda / 2$ plate and a following generator cell filled with $\mathrm{CCl}_{4}$. Stokes light $E_{\mathrm{S}}$ with a frequency shift of $\approx 459 \mathrm{~cm}^{-1}$ is generated by transient stimulated scattering with a conversion efficiency of approximately $10^{-2}$. The laser pulse and the generated Stokes pulse traverse a polarizer $\mathrm{P} 1$ and are imaged into the sample cell $(l=1 \mathrm{~cm})$. Both pulses produce a coher- 


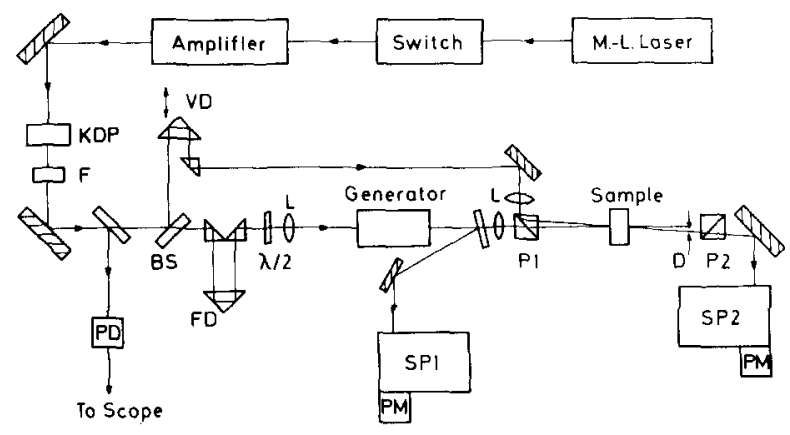

Fig. 2. Schematic of the experimental system to measure the coherent probe scattering of a resonant and a nonresonant susceptibility $\chi^{(3)}$. Beamsplitter, BS; variable and fixed delay, $\mathrm{VD}$ and $\mathrm{FD} ; \lambda / 2$ plate, $\lambda / 2$; lenses, $L$; polarizers, $\mathrm{P} 1$ and $\mathrm{P} 2$; aperture, D; spectrometers, SP1 and SP2; photomultipliers, PM; photodiode, PD.

ent material excitation in the sample. The probing light pulse $E_{\mathrm{L} 2}$ of smaller intensity is generated by the beam splitter BS and is properly delayed by the variable delay VD. The probe field is polarized perpendicular to the excitation fields; it crosses the excitation beam in the sample under a small angle of $7 \mathrm{mrad}$. The scattered Stokes light passes the aperture $\mathrm{D}$, a second polarizer $\mathrm{P} 2$, and is detected by the photomultiplier PM.

\section{Results and discussion}

In a first experiment we investigate coherent probe scattering of pure $\mathrm{CCl}_{4}$ under resonant excitation; the contribution of $\chi_{N R}$ is negligible (fig. 3, curve 1). The totally symmetric tetrahedron vibration is measured. This mode shows isotopic line splitting. In the spontaneous Raman spectrum [10] four components can be resolved. While all components are excited by the pumping processes, only the most abundant species at $459 \mathrm{~cm}^{-1}$ contributes to the observed scattering signal. This fact results from the selective $k$-matching geometry chosen in our experiments adjusting $k$-matching only for the central line [11].

The observed coherent signal $S^{\text {coh }}$ is plotted in fig. 3, curve 1 , as a function of delay time $t_{\mathrm{D}}$, where $t_{\mathrm{D}}=0$ marks the maximum of the pumping laser pulse. The experimental points represent average values of approximately 15 individual measurements. The observed data show the predicted behavior for Raman probe scattering: A delayed maximum and an exponential decay

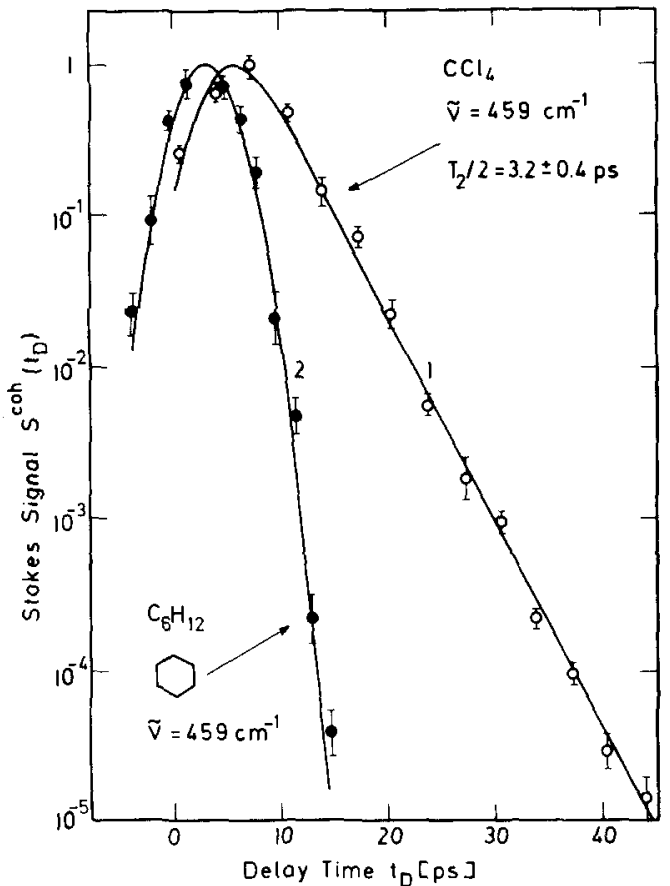

Fig. 3. Normalized coherent probe scattering signal versus delay time $t_{D}$ in neat liquids. In case 1 , the vibrational mode in $\mathrm{CCl}_{4}$ at $459 \mathrm{~cm}^{-1}$ was excited resonantly. We observe a dephasing time of $3.2 \mathrm{ps}$. Curve 2 shows the case of nonresonant excitation in $\mathrm{C}_{6} \mathrm{H}_{12}$ with $\widetilde{\nu}=\widetilde{\nu}_{\mathrm{L}}-\widetilde{\nu}_{\mathrm{S}}=459 \mathrm{~cm}^{-1}$. The solid lines are calculated for a gaussian input pulse with duration $t_{\mathrm{p}}=5.2 \mathrm{ps}$.

with a time constant of $T_{2} / 2=3.2 \pm 0.4 \mathrm{ps}$ for the neat liquid. This value of $T_{2}$ is in accordance with previous measurements where different probing geometries were used in a Raman generator set-up [10].

Next, we discuss our results on liquid cyclohexane, $\mathrm{C}_{6} \mathrm{H}_{12}$. This substance was chosen since it has no vibrational transitions in the frequency range around 459 $\mathrm{cm}^{-1}$, i.e. a non-resonant excitation is investigated. Fig. 3, curve 2, presents our probe scattering data obtained with the same excitation pulses and the identical experimental geometry as used in the preceding experiment. The scattered signal rapidly increases to a maximum and then displayes a steep non-exponential decay. The theoretical line in the figure was calculated for an incident laser pulse of gaussian shape with a duration $t_{\mathrm{p}}=5.2 \mathrm{ps}$. The good agreement with the experimental points extends over a range of five orders of magnitude. This result gives clear evidence that the experimental pulse shape is indeed close to gaussian function. 
We measured the ratio of the maxima of the two curves of fig. 3 and found $S_{\max }^{\text {coh }}\left(\mathrm{CCl}_{4}\right) / S_{\max }^{\mathrm{coh}}\left(\mathrm{C}_{6} \mathrm{H}_{12}\right) \approx$ 24 . This value is estimated to be accurate to $30 \%$. Using the known value of the stimulated Raman gain of $g=1.3 \mathrm{~cm} / \mathrm{MW}[11]^{\ddagger}$ we deduce the nonresonant susceptibility in $\mathrm{C}_{6} \mathrm{H}_{12}$ to be $\chi_{z y z y}^{(3)}\left(-\omega_{\mathrm{S}} ; \omega_{\mathrm{S}}, \omega_{\mathrm{L}},-\omega_{\mathrm{L}}\right)=(1.5 \pm 0.6) \times 10^{-14} \mathrm{esu}$.

The value is of the order of magnitude expected from other nonlinear measurements $[16,17]$.

The role of nonresonant and resonant contributions to coherent probe scattering is readily seen in mixed systems. Fig. 4 presents data of a solution of $\mathrm{CCl}_{4}(9 \%$ volume in $\mathrm{C}_{6} \mathrm{H}_{12}$ ). It is interesting to see that our ex-

* The gain factor is corrected for the neighboring isotopic species of $\mathrm{CCl}_{4}$ which do not contribute to the coherent signal.

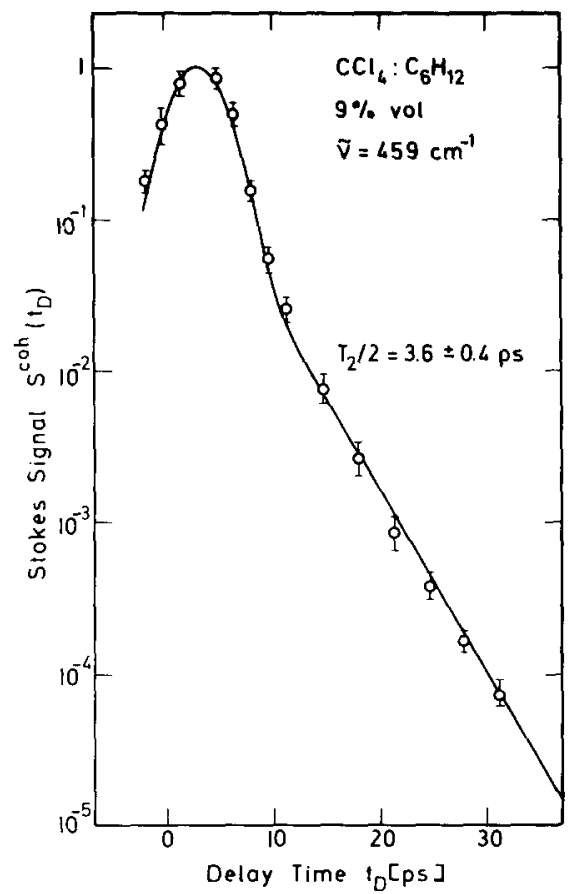

Fig. 4. Coherent probe scattering signal versus delay time measured in a mixture of $\mathrm{CCl}_{4}: \mathrm{C}_{6} \mathrm{H}_{12}$ (9:91 by volume). Same excitation as in fig. $3\left(\widetilde{\nu}=459 \mathrm{~cm}^{-1}\right)$. The solid line is calculated with $\times_{\mathrm{NR}} / N \partial \alpha / \partial q=0.66$ and $T_{2} / 2=3.6 \mathrm{ps}$. The maximum near $t \mathrm{D}=0$ is due to the nonresonant $\chi^{(3)}$ of the cyclohexane molecules. The exponential decay for $t_{\mathrm{D}}>10 \mathrm{ps}$ corresponds to the resonant contributions of the $\mathrm{CCl}_{4}$ molecules. perimental data show the behavior expected from the theoretical discussion presented in context with fig. 1 . A rapid rise to a maximum and subsequent steep decay are due to the nonresonant contribution to $S^{\operatorname{coh}}\left(t_{\mathrm{D}}\right)$. For larger values of $t_{\mathrm{D}}$ the signal decay slows down and reaches an exponential asymptote representing the resonant contribution to $S^{\text {coh }}$. From the exponential slope starting a factor of $\approx 100$ below the maximum the dephasing time of $\mathrm{CCl}_{4}$ is directly determined. We find a $T_{2} / 2=3.6 \pm 0.4$ in the mixture with $\mathrm{C}_{6} \mathrm{H}_{12}$. Comparing this number with our result of $T_{2}$ in the neat liquid (fig. 3) suggests a small increase of approximately $10 \%$. The difference is not surprising since concentration dependent relaxation processes may contribute to $T_{2}$, e.g. resonant energy transfer between neighboring $\mathrm{CCl}_{4}$ molecules.

The curve in fig. 4 is calculated from eqs. (2) and (6) using the experimental value of $T_{2} / 2=3.6 \mathrm{ps}$; the ratio of $\chi_{N R} / N(\partial \alpha / \partial q)$ is the only fitting parameter. The calculated curve accounts well for the experimental points. The obtained value of $\chi_{\mathrm{NR}}$ is consistent with the number quoted above within a factor of approximately 1.5 . The agreement is satisfactory considering the uncertainties of the gain factor of $\mathrm{CCl}_{4}$ in $\mathrm{C}_{6} \mathrm{H}_{12}$ and the small solvent shift of the Raman transition $\left(\$ 0.2 \mathrm{~cm}^{-1}\right)$.

In conclusion we wish to emphasize that coherent probe scattering offers the possibility to study nonresonant and resonant parts of the third order susceptibility. The different time behavior of both contributions has been observed in our measurements for the first time. Our experimental system allows to study liquid mixtures in a large concentration range yielding information on the magnitude of the non-resonant susceptibility relative to Raman scattering data. In addition, relaxation processes and intermolecular interaction in diluted systems may be investigated.

\section{References}

[1] P.D. Maker and R.W. Terhune, Phys. Rev. 137 (1965) A801.

[2] W.G. Rado, Appl. Phys. Lett. 11 (1967) 123.

[3] M.D. Levenson, C. Flyztanis and N. Bloembergen, Phys. Rev. 6 (1972) 3462;

M.D. Levenson and N. Bloembergen, Phys. Rev. B10 (1974) 4447. 
[4] P.R. Regnier and J.P.E. Taran, Appl. Phys. Lett. 23 (1973) 240.

[5] D. Heiman, R.W. Hellwarth, M.D. Levenson and G. Martin, Phys. Rev. Lett. 36 (1976) 189;

M.D. Levenson and J.J. Song, J. Opt. Soc. Am. 66 (1976) 641.

[6] A. Penzkofer and W. Kaiser, Opt. Quant. Electron 9 (1977) 315 .

[7] D. Milam, M.J. Weber and A.J. Glass, Appl. Phys. Lett. 31 (1977) 822 .

[8] W. Zinth, A. Laubereau and W. Kaiser, to be published.

[9] D. von der Linder, A. Laubereau and W. Kaiser, Phys. Rev. Lett. 26 (1971) 954.

[10] A. Laubereau, G. Wochner and W. Kaiser, Phys. Rev. A13 (1976) 2212.
[11] For a review see: A. Laubereau and W. Kaiser, Rev. Mod. Phys., to be published.

[12] A. Laobereau, G. Wochner and W. Kaiser, Chem. Phys. 28 (1978) 363.

[13] J.A. Giordmaine and W. Kaiser, Phys. Rev. 144 (1966) 676.

[14] R.L. Carman, F. Shimizu, C.S. Wang and N. Bloembergen, Phys. Rev. A2 (1970) 60;

S.A. Akhmanov, K.N. Brabovich, A.P. Sukhorukov and A.S. Chirikin, Sov. Phys. JETP 32 (1971) 266.

[15] W. Zinth, A. Laubereau and W. Kaiser, Opt. Commun. 22 (1977) 161.

[16] M. Paillette, Ann. Phys. (Paris) 4 (1969) 671.

[17] R.W. Hellwarth, Prog. Quant. Electron 5 (1977) 1. 